Check for updates

Cite this: J. Mater. Chem. A, 2019, 7, 15054

Received 16th March 2019 Accepted 29th May 2019

DOI: $10.1039 / c 9 t a 02884 f$

rsc.li/materials-a

\section{In situ fabricated nickel vanadate/N-doped reduced graphene oxide hybrid as an advanced electrocatalyst in alkaline hydrogen evolution reaction $\dagger$}

\author{
Ayon Karmakar (D) and Suneel Kumar Srivastava (D) *ab
}

\begin{abstract}
Inthe current work, we reported the in situ fabrication of a nickel vanadate $\left(\mathrm{Ni}_{3} \mathrm{~V}_{2} \mathrm{O}_{8}\right)$-anchored $\mathrm{N}$-doped reduced graphene oxide (NRGO) hybrid by a simple one-step reflux method. Subsequently, the electrocatalytic performance of the $\mathrm{Ni}_{3} \mathrm{~V}_{2} \mathrm{O}_{8} / \mathrm{NRGO}$ hybrid was investigated in an alkaline medium $(1.0 \mathrm{M} \mathrm{KOH})$ for the hydrogen evolution reaction (HER). It was noted that the optimization of the NRGO content ( $5.6 \mathrm{wt} \%$ ) highly influenced the homogeneous distribution of quasi-spherical $\mathrm{Ni}_{3} \mathrm{~V}_{2} \mathrm{O}_{8}$ nanoparticles over NRGO sheets and enhanced the water reduction ability. This hybrid material exhibited a sufficiently high electrochemical active surface area (517.5 $\mathrm{cm}^{2}$ ) and remarkably low charge transfer resistance $(\sim 1.6 \Omega)$. Furthermore, the very low overpotential $(\sim 43 \mathrm{mV})$ and the high exchange current density $\left(\sim 1.24 \mathrm{~mA} \mathrm{~cm}{ }^{-2}\right)$ of $\mathrm{Ni}_{3} \mathrm{~V}_{2} \mathrm{O}_{8} / \mathrm{NRGO}$ (5.6 wt\%) demonstrated its promising HER performance. Additionally, superior long-term and accelerated stability compared to that of the benchmark $\mathrm{Pt} / \mathrm{C}$ in a strong basic medium clearly signified that $\mathrm{Ni}_{3} \mathrm{~V}_{2} \mathrm{O}_{8} / \mathrm{NRGO}$ (5.6 wt\%) can act as an efficient, cost-effective, and durable electrocatalyst for water electrolyzers.
\end{abstract}

Hydrogen is one of the most important alternative fuels compared to many conventional energy resources. ${ }^{1}$ In this regard, the electrolysis of water in an alkaline medium remains the most cost-effective, sustainable and industrially accepted method for hydrogen production. ${ }^{2}$ However, the high overpotential of the hydrogen evolution reaction (HER) affects the efficiency of hydrogen generation. ${ }^{3}$ Therefore, it is mandatory to lower the overpotential of HER in the presence of suitable electrocatalyst(s). As a result, platinum (Pt) has been reported to be the most active HER electrocatalyst of recent times. ${ }^{4}$

anorganic Materials and Nanocomposite Laboratory, Department of Chemistry, Indian Institute of Technology Kharagpur, Kharagpur 721302, India. E-mail: sunil111954@yahoo.co.uk; Tel: +91-3222-283334

${ }^{b}$ School of Energy Science and Engineering, Indian Institute of Technology Kharagpur, Kharagpur 721302, India

$\dagger$ Electronic supplementary information (ESI) available: Experimental section, characterization techniques, supplementary figures and tables are provided. See DOI: $10.1039 / \mathrm{c} 9 \mathrm{ta} 02884 \mathrm{f}$
However, the widespread use of Pt for industrialization and commercialization is limited due to its scarcity, very high cost and inferior durability in strong alkaline solutions. ${ }^{5}$ According to the available literature, mixed transition metal oxides compared to the corresponding monometallic oxides show better electrochemical activity due to the presence of a large number of active sites. ${ }^{6,7}$ In this regard, transition metal vanadates, especially $\mathrm{Ni}_{3} \mathrm{~V}_{2} \mathrm{O}_{8}, \mathrm{Co}_{3} \mathrm{~V}_{2} \mathrm{O}_{8}$, and $\mathrm{CoVO}_{x}$, exhibit enhanced electrochemical activity owing to the multiple valence oxidation states of vanadium. ${ }^{8-11}$ In view of this, $\mathrm{Ni}_{3} \mathrm{~V}_{2} \mathrm{O}_{8}$ nanowires grown on a Ti-foil, ${ }^{12} \mathrm{Ni}_{3} \mathrm{~V}_{2} \mathrm{O}_{8}$ /carbon cloth, ${ }^{8}$ urchinshaped $\mathrm{Ni}_{3}\left(\mathrm{VO}_{4}\right)_{2}$ hollow nanospheres, ${ }^{13}$ nickel vanadate nanopetals, ${ }^{14}$ and $\mathrm{Ni}_{3}\left(\mathrm{VO}_{4}\right)_{2} / \mathrm{rGO}$ nanocomposites ${ }^{\mathbf{1 5}}$ have been successfully investigated for their applications in lithium-ion batteries, sodium-ion batteries, and supercapacitors. However, there exists very limited literature related to the application of $\mathrm{Ni}_{3} \mathrm{~V}_{2} \mathrm{O}_{8}$ in electrocatalytic energy conversions. Shang et al. ${ }^{\mathbf{1 6}}$ observed a moderate overpotential of $\sim 113 \mathrm{mV}$ at $10 \mathrm{~mA} \mathrm{~cm}^{-2}$ current density for HER in a 1.0 M KOH solution in the nickel foam-supported combination of two mixed transition metal oxides $\left(\mathrm{Ni}_{3}\left(\mathrm{VO}_{4}\right)_{2} @ \mathrm{NiCo}_{2} \mathrm{O}_{4} / \mathrm{NF}\right)$. In addition, sea-urchin-like $\mathrm{Ni}_{3}\left(\mathrm{VO}_{4}\right)_{2}$ nanostructures showed promising electrocatalytic HER activity with a very small overpotential $\left(\eta_{10}\right)$ of about $90 \mathrm{mV}$ at $10 \mathrm{~mA} \mathrm{~cm}{ }^{-2}$ and a small Tafel slope $\left(50 \mathrm{mV} \mathrm{dec}^{-1}\right)$ in $0.5 \mathrm{M}$ $\mathrm{H}_{2} \mathrm{SO}_{4} \cdot{ }^{17}$ However, the low charge transfer resistance and stability of electrocatalysts (non-precious) in a strong alkaline medium are few other desirable parameters to be taken into consideration for their superior electrocatalytic performance., ${ }^{\mathbf{4} 18}$

Motivated by these facts, we focused our work on the fabrication of a nickel vanadate $\left(\mathrm{Ni}_{3} \mathrm{~V}_{2} \mathrm{O}_{8}\right)$ nanoparticle-decorated nitrogen-doped RGO (NRGO) hybrid through a simple onestep reflux method (ESI $\dagger$ ). Scheme 1 clearly demonstrates the in situ fabrication of the $\mathrm{Ni}_{3} \mathrm{~V}_{2} \mathrm{O}_{8} / \mathrm{NRGO}$ hybrids. The choice of NRGO in the present work was mainly guided by its high electrical conductivity and the presence of larger defects. Therefore, it is anticipated that the structural stability of the electrode material can be enhanced in the presence of NRGO as the conducting backbone in a strong alkaline medium. Notably, to 


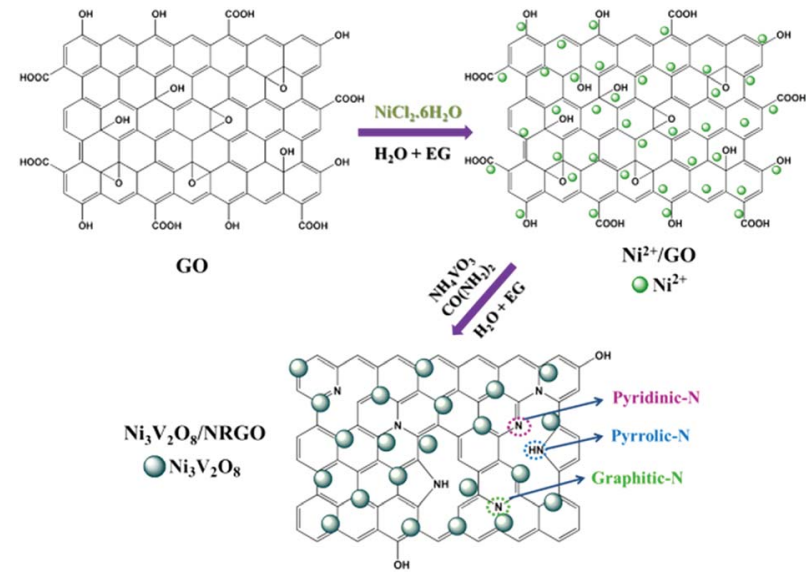

Scheme 1 Probable formation pathway of $\mathrm{Ni}_{3} \mathrm{~V}_{2} \mathrm{O}_{8} / \mathrm{NRGO}$ hybrids.

the best of our knowledge, no reports are available on evaluating the electrocatalytic HER performance of an $\mathrm{Ni}_{3} \mathrm{~V}_{2} \mathrm{O}_{8}$ / NRGO hybrid in an alkaline solution. In this context, our findings showed that the $\mathrm{Ni}_{3} \mathrm{~V}_{2} \mathrm{O}_{8} / \mathrm{NRGO}(\sim 5.6 \mathrm{wt} \%)$ hybrid demonstrated superior HER activity in an alkaline medium.

The formation pathway of the $\mathrm{Ni}_{3} \mathrm{~V}_{2} \mathrm{O}_{8} / \mathrm{NRGO}$ hybrids through a simple one-step reflux method, as displayed in Scheme 1, consists of the adsorption of $\mathrm{Ni}^{2+}$ ions on the surface of graphene oxide (GO). This is most likely due to the electrostatic attraction between the positively charged metal ions $\left(\mathrm{Ni}^{2+}\right)$ and the negatively charged oxygen functionalities $(-\mathrm{COOH}$, $-\mathrm{OH})$ of $\mathrm{GO}$ (Fig. S2 $\dagger$ ). Subsequently, the solutions of $\mathrm{NH}_{4} \mathrm{VO}_{3}(2$ $\mathrm{mmol}$ ) and urea $(12 \mathrm{mmol}$ ) prepared in a mixed solvent (water + EG) were added to the earlier dispersion of $\mathrm{Ni}^{2+} / \mathrm{GO}$. As a result, $\mathrm{NH}_{3}$ (source of nitrogen) was formed during the hydrolysis and the transesterification of urea in the presence of water and ethylene glycol, respectively. ${ }^{19}$ In addition, $\mathrm{Ni}^{2+}$ and $\mathrm{VO}_{3}{ }^{-}$ions originating from $\mathrm{NiCl}_{2} \cdot 6 \mathrm{H}_{2} \mathrm{O}$ and $\mathrm{NH}_{4} \mathrm{VO}_{3}$ combined together to form $\mathrm{Ni}_{3} \mathrm{~V}_{2} \mathrm{O}_{8}$ in the basic medium. Furthermore, ammonia generated during the hydrolysis and the transesterification of urea successfully reduced $\mathrm{GO}$ to RGO, followed by simultaneous $\mathrm{N}$ doping to form the $\mathrm{Ni}_{3} \mathrm{~V}_{2} \mathrm{O}_{8} / \mathrm{NRGO}$ hybrid. The plausible reactions involved in the fabrication of the $\mathrm{Ni}_{3} \mathrm{~V}_{2} \mathrm{O}_{8}$ / NRGO hybrid can be summarized as follows: $:^{20,21}$

$$
\begin{gathered}
\mathrm{Ni}^{2+}+\mathrm{GO} \rightarrow \mathrm{Ni}^{2+} / \mathrm{GO} \\
\mathrm{CO}\left(\mathrm{NH}_{2}\right)_{2}+\mathrm{H}_{2} \mathrm{O} \rightarrow 2 \mathrm{NH}_{3}+\mathrm{CO}_{2} \\
\mathrm{CO}\left(\mathrm{NH}_{2}\right)_{2}+\mathrm{HO}-\mathrm{CH}_{2} \mathrm{CH}_{2}-\mathrm{OH} \rightarrow\left(\mathrm{CH}_{2} \mathrm{O}\right)_{2} \mathrm{CO}+2 \mathrm{NH}_{3} \\
\mathrm{NH}_{3}+\mathrm{H}_{2} \mathrm{O} \rightarrow \mathrm{NH}_{4}^{+}+\mathrm{OH}^{-} \\
3 \mathrm{Ni}^{2+} / \mathrm{GO}+2 \mathrm{VO}_{3}^{-}+4 \mathrm{OH}^{-} \stackrel{\mathrm{NH}_{4}^{+}}{\longrightarrow} \mathrm{Ni}_{3} \mathrm{~V}_{2} \mathrm{O}_{8} / \mathrm{NRGO}+2 \mathrm{H}_{2} \mathrm{O}
\end{gathered}
$$

Thermogravimetric analysis (TGA) in air revealed that the NV/NRGO1, NV/NRGO2, and NV/NRGO3 hybrids contained $\sim 3.3,5.6$, and $6.6 \mathrm{wt} \%$ NRGO, respectively (ESI $\dagger$ ). Furthermore, the results of the CHN analyses of the hybrids were also in agreement with the TGA results (Table S1 $\dagger$ ). The X-ray diffraction (XRD) patterns of the NV, NRGO, and NV/NRGO hybrids were obtained and the corresponding findings are displayed in Fig. 1a. The XRD pattern of NRGO showed the appearance of peaks at $\sim 23.8^{\circ}(002)$ and $\sim 43.0^{\circ}(100)$ similar to that reported for reduced graphene oxide. ${ }^{22}$ It is also evident from Fig. 1a that the XRD patterns of NV as well as NV/NRGO hybrids show the formation of the pure orthorhombic phase (JCPDS no. 74-1484) of $\mathrm{Ni}_{3} \mathrm{~V}_{2} \mathrm{O}_{8}$ without any impurity peaks corresponding to $\mathrm{NiO}$ or $\mathrm{V}_{2} \mathrm{O}_{5}$. Interestingly, the intensity of the NRGO peaks almost disappeared for NV/NRGO hybrids, indicating the possible formation of $\mathrm{Ni}_{3} \mathrm{~V}_{2} \mathrm{O}_{8}$ on highly exfoliated NRGO sheets. This could be further ascertained by the morphological investigations of the NV/NRGO hybrids through electron microscopy. The Raman spectra (Fig. 1b) show that the peaks appearing at $760 \mathrm{~cm}^{-1}$ (low intensity) and $823 \mathrm{~cm}^{-1}$ (high intensity) for NV correspond to the asymmetric and symmetric stretching vibration modes of the $\mathrm{V}-\mathrm{O}$ bonds. ${ }^{15,17}$ However, the intensity of the peak at $823 \mathrm{~cm}^{-1}$ is considerably reduced for all the hybrids, while the peak corresponding to $\sim 760 \mathrm{~cm}^{-1}$ almost disappears for the NV/NRGO3 hybrids compared to that for NV. The Raman spectra of the NV/NRGO hybrids also exhibit the presence of D $\left(\sim 1596 \mathrm{~cm}^{-1}\right)$ and $\mathrm{G}\left(\sim 1359 \mathrm{~cm}^{-1}\right)$ bands appearing at nearly identical positions. This finding suggests that the graphitic structure remains unaltered in the hybrids. ${ }^{23}$ Furthermore, the $I_{\mathrm{D}} / I_{\mathrm{G}}$ ratios corresponding to the NV/NRGO hybrids were calculated and followed the order NV/NRGO2 $(0.86)>\mathrm{NV} /$ NRGO1 (0.84) > NV/NRGO3 (0.76). The relatively higher value of the $I_{\mathrm{D}} / I_{\mathrm{G}}$ ratio in NV/NRGO2 could be ascribed to the presence of higher defects/disorders. ${ }^{24}$

The X-ray photoelectron spectroscopy (XPS) survey scans of $\mathrm{NV}$ and NV/NRGO2 are displayed in Fig. 2a. Furthermore, the high-resolution Ni 2p and V 2p spectra of NV were compared with that of NV/NRGO2 (Fig. S4†). It was noted that the Ni $2 p$ and $\mathrm{V} 2 \mathrm{p}$ peaks present in NV/NRGO2 shifted to higher binding energies with respect to that for NV due to the strong electronic interaction between $\mathrm{Ni}_{3} \mathrm{~V}_{2} \mathrm{O}_{8}$ and NRGO present in NV/NRGO2. Such interaction between $\mathrm{C} / \mathrm{N}(\mathrm{NRGO})$ and $\mathrm{Ni} / \mathrm{V}\left(\mathrm{Ni}_{3} \mathrm{~V}_{2} \mathrm{O}_{8}\right)$ that leads to an increase in the respective binding energies can be accounted on the basis of the reduction in the electron density around $\mathrm{Ni} / \mathrm{V}$ in $\mathrm{NV} / \mathrm{NRGO} 2$ as a result of their electronegativity $(\chi)$ differences $\left(\chi_{\mathrm{Ni}}=1.91, \chi_{\mathrm{V}}=1.63, \chi_{\mathrm{C}}=2.55, \chi_{\mathrm{N}}=3.04\right) .{ }^{25}$ The survey spectrum (Fig. $2 \mathrm{a}$ ) of NV/NRGO2 clearly shows the
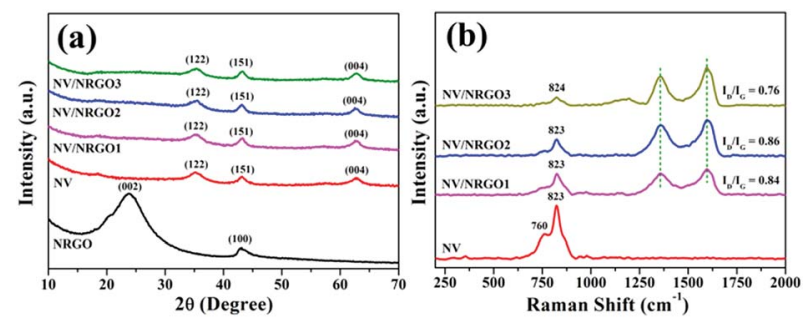

Fig. 1 XRD patterns (a) and Raman spectra (b) of NRGO, NV, NV/ NRGO1, NV/NRGO2, and NV/NRGO3. 

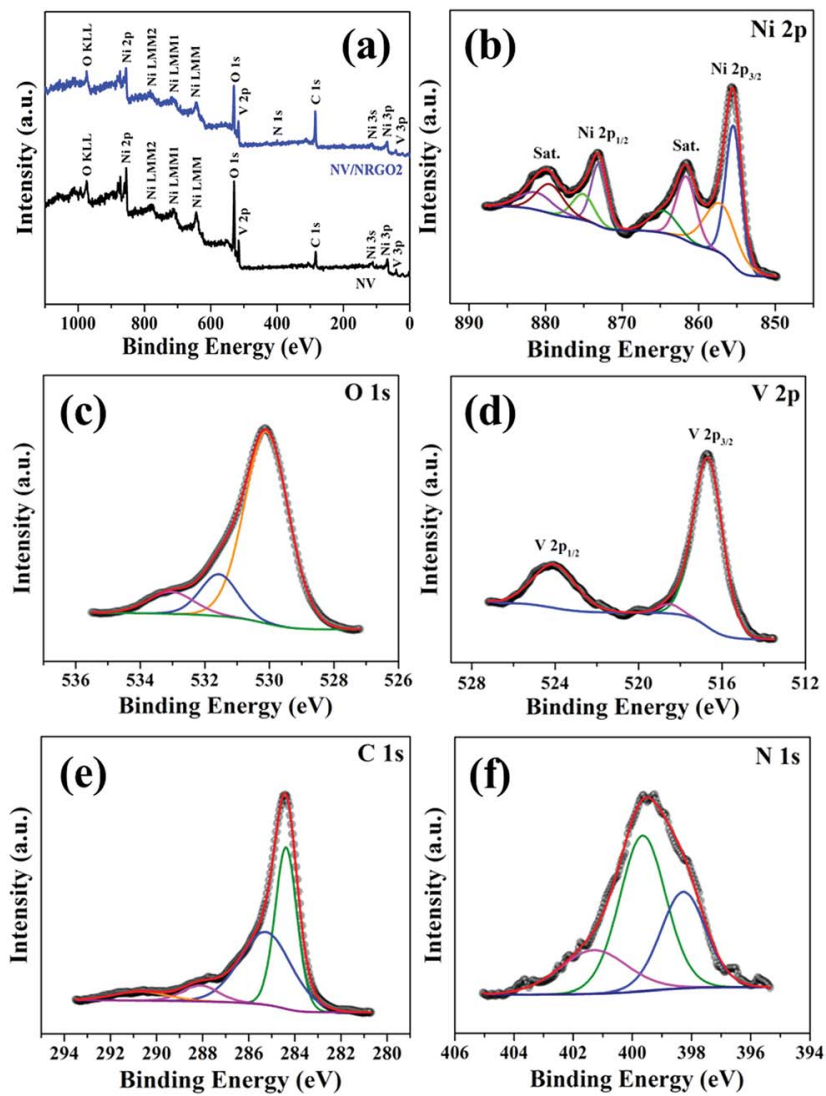

Fig. 2 (a) XPS survey scans of NV and NV/NRGO2 and deconvoluted high-resolution XPS spectra of (b) Ni 2p, (c) O 1s, (d) $\vee 2 p$, (e) C 1s, and (f) $\mathrm{N}$ 1s of NV/NRGO2.

presence of $\mathrm{Ni}, \mathrm{V}, \mathrm{O}, \mathrm{C}$, and $\mathrm{N}$. Fig. $2 \mathrm{~b}-\mathrm{f}$ show the highresolution core levels and the corresponding deconvoluted spectra of Ni 2p, O 1s, V 2p, C 1s, and N 1s, respectively. The peaks at $873.0 \mathrm{eV}\left(\mathrm{Ni} 2 \mathrm{p}_{1 / 2}\right)$ and $855.4 \mathrm{eV}\left(\mathrm{Ni} 2 \mathrm{p}_{3 / 2}\right)$ in $\mathrm{Ni} 2 \mathrm{p}$ spectra correspond to the presence of $\mathrm{Ni}^{2+}$ in NV/NRGO2..$^{16,17}$ This is also supplemented by the presence of satellite peaks at 881.2 and $861.6 \mathrm{eV} \cdot{ }^{16} \mathrm{In}$ addition, the peaks at $875.1 \mathrm{eV}\left(\mathrm{Ni} 2 \mathrm{p}_{1 / 2}\right)$ and $857.1 \mathrm{eV}\left(\mathrm{Ni} 2 \mathrm{p}_{3 / 2}\right)$ can be assigned to the existence of $\mathrm{Ni}^{3+}{ }^{36}$ In the case of $\mathrm{O} 1 \mathrm{~s}$, the peaks at $\sim 530.1,531.6$, and $533.0 \mathrm{eV}$ correspond to the metal-oxygen bond, oxygen vacancies, and surface -OH groups. ${ }^{15}$ Furthermore, the peaks at $\sim 524.0,518.5$, and $516.7 \mathrm{eV}$ in the $\mathrm{V} 2 \mathrm{p}$ spectrum can be attributed to the +5 oxidation state of $\mathrm{V} .^{17,27}$ The intense peak at $284.4 \mathrm{eV}$ in the $\mathrm{C} 1 \mathrm{~s}$ spectrum corresponds to $\mathrm{sp}^{2}$ hybridized graphitic $\mathrm{C}$ originating from NRGO. ${ }^{4}$ The other additional peaks at $\sim 290.7,288.1$, and $285.3 \mathrm{eV}$ can be assigned to $\mathrm{C}=\mathrm{O}, \mathrm{N}-\mathrm{sp}^{3} \mathrm{C}$, and $\mathrm{N}-\mathrm{sp}^{2} \mathrm{C}$, respectively. ${ }^{15}$ The peaks in the spectrum of $\mathrm{N} 1 \mathrm{~s}$ can be indexed to graphitic-N (401.3 eV), pyrrolic-N (399.6 eV), and pyridinic- $\mathrm{N}$ $(398.3 \mathrm{eV}) .{ }^{4}$ These findings clearly demonstrate the nitrogen doping ( 1.6 at\%) of RGO and the formation of highly pure $\mathrm{Ni}_{3} \mathrm{~V}_{2} \mathrm{O}_{8} / \mathrm{NRGO}$ hybrids. Also, the presence of pyrrolic- $\mathrm{N}$ is responsible for the high electrical conductivity of NRGO, while pyridinic-N facilitates electrocatalytic activity. ${ }^{28}$ Therefore, the optimised NRGO content in NV/NRGO2 is most likely to enhance the electrocatalytic HER performance of $\mathrm{Ni}_{3} \mathrm{~V}_{2} \mathrm{O}_{8}$.
Field emission scanning electron microscopy (FESEM) and transmission electron microscopy (TEM) were employed to reveal the formation of the aggregated sub-micrometre hierarchical spherical particles of NV (Fig. 3a and e) and the distribution of $\mathrm{Ni}_{3} \mathrm{~V}_{2} \mathrm{O}_{8}$ particles on NRGO sheets for the NV/NRGO hybrids (Fig. $3 \mathrm{~b}-\mathrm{h}$ ). It was clearly seen that the addition of NRGO resulted in a narrow size and better distribution of the $\mathrm{Ni}_{3} \mathrm{~V}_{2} \mathrm{O}_{8}$ particles on the NRGO sheets compared to that for pure NV. Furthermore, a lower degree of exfoliation of NRGO in the NV/NRGO1 hybrid was observed (Fig. $3 \mathrm{~b}$ and f). It was noted that the agglomerated $\mathrm{Ni}_{3} \mathrm{~V}_{2} \mathrm{O}_{8}$ particles covered entire NRGO sheets and bare sheets of NRGO were hardly visible. When the amount of GO was increased during the preparation of the $\mathrm{NV} /$ NRGO2 hybrid, relatively better exfoliation of NRGO and uniform distribution of $\mathrm{Ni}_{3} \mathrm{~V}_{2} \mathrm{O}_{8}$ particles were observed over the NRGO sheets, as displayed in Fig. $3 \mathrm{c}$ and g. The FESEM/TEM images of the NV/NRGO3 hybrid in Fig. 3d and h indicate the irregular distribution of $\mathrm{NV}$ particles on NRGO sheets due to their self-agglomeration with the visible bare surface of NRGO, indicating its incomplete coverage. Considering all these observations, it is anticipated that the optimization of the $\mathrm{GO} /$

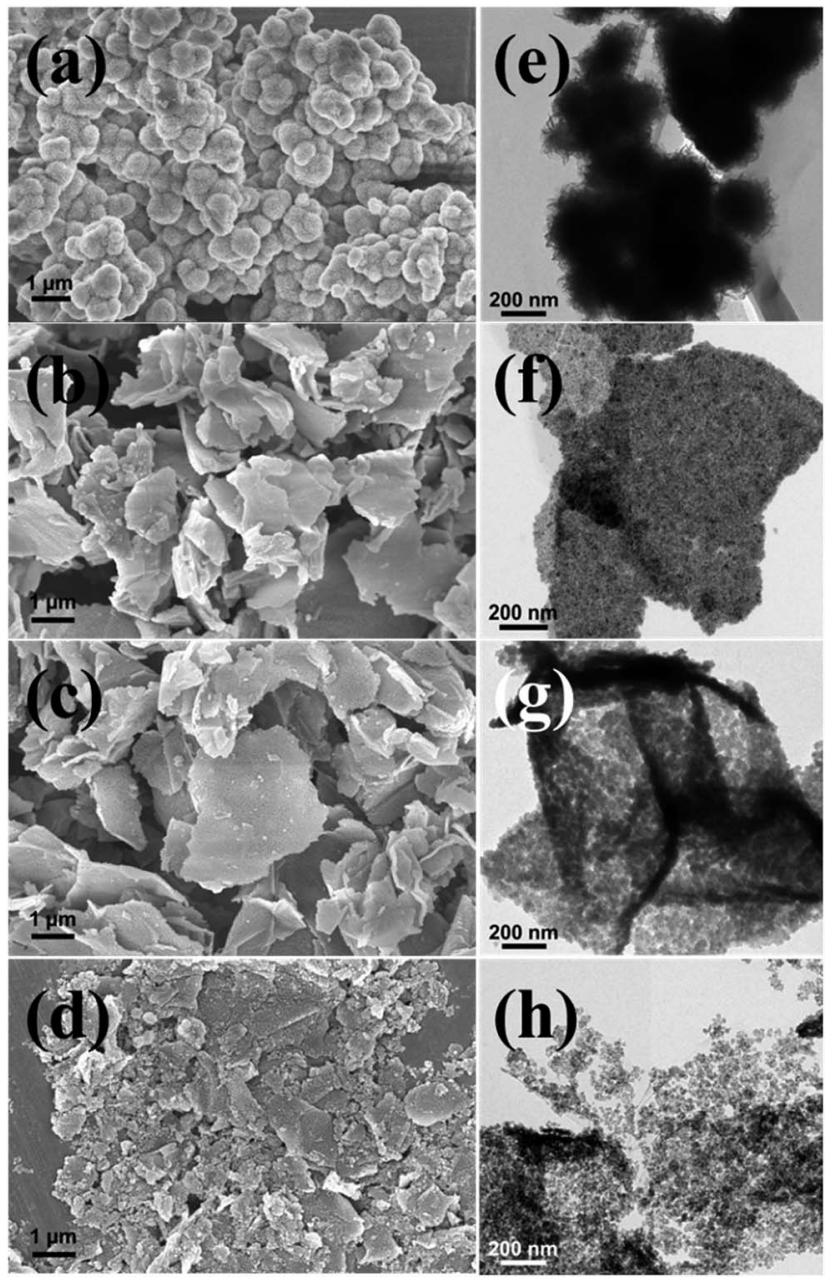

Fig. 3 FESEM images $(\mathrm{a}-\mathrm{d})$ and corresponding TEM images (e-h) of $\mathrm{NV}, \mathrm{NV} / \mathrm{NRGO} 1, \mathrm{NV} / \mathrm{NRGO} 2$, and NV/NRGO3. 
$\mathrm{Ni}_{3} \mathrm{~V}_{2} \mathrm{O}_{8}$ ratio in the fabrication of the NV/NRGO hybrids is the key factor to achieve uniform distribution of the NV particles on the NRGO sheets. Furthermore, the high-magnification FESEM and TEM images (Fig. S5a and $\mathrm{b} \dagger$ ) of the NV/NRGO2 hybrid also reaffirmed our earlier contention on the homogeneous distribution of the $\mathrm{Ni}_{3} \mathrm{~V}_{2} \mathrm{O}_{8}$ nanoparticles on the NRGO sheets. The corresponding HRTEM image of the NV/NRGO2 hybrid is also displayed in Fig. S5c. $\dagger$ It clearly indicates the growth of the (122) plane $(d=0.249 \mathrm{~nm})$, and the presence of concentric rings in the selected area diffraction pattern (SAED) (in the inset of Fig. S5c $\dagger$ ) demonstrates its polycrystalline nature. The elemental composition analysis of the marked region of $\mathrm{NV} /$ NRGO2 in Fig. S5d $\dagger$ was carried out through STEM-elemental mapping and the results are displayed in Fig. S5(e-h), $\dagger$ which clearly demonstrate the presence of $\mathrm{Ni}, \mathrm{V}, \mathrm{O}$, and $\mathrm{N}$ in $\mathrm{NV} /$ NRGO2. It may be noted that the mapping of $\mathrm{C}$ was not taken into account due to its presence in the carbon-coated copper grid. The energy dispersive X-ray (EDX) analysis of NV/NRGO2 in Fig. S6 $\dagger$ also confirms that the Ni-to-V atomic ratio is $\sim 3: 2$, indicating the formation of $\mathrm{Ni}_{3} \mathrm{~V}_{2} \mathrm{O}_{8}$, as observed from the XRD analysis (Fig. 1a). In addition, the Ni-to-V molar ratio ( 1.59) obtained from the ICP-MS analysis (Table S2 $\dagger$ ) confirmed our observations obtained from EDX.

The electrocatalytic HER polarization curves of Pt/C (20 wt\%) and the NV, NRGO, NV/RGO and NV/NRGO hybrids (scan rate: $5 \mathrm{mV} \mathrm{s}^{-1}$ ) in $1.0 \mathrm{M} \mathrm{KOH}$ are displayed in Fig. 4a. The overpotentials $(\eta)$ required to achieve $10 \mathrm{~mA} \mathrm{~cm}^{-2}$ cathodic current density were found to be $\sim 16,97,194,236,94,43$, and $81 \mathrm{mV}$ for $\mathrm{Pt} / \mathrm{C}, \mathrm{NV}, \mathrm{NRGO}, \mathrm{NV} / \mathrm{RGO}, \mathrm{NV} / \mathrm{NRGO} 1, \mathrm{NV} / \mathrm{NRGO} 2$, and NV/ NRGO3, respectively. Our investigations on the HER activity of $\mathrm{NV}\left(\eta_{10}=97 \mathrm{mV}\right)$ and NRGO sheets $\left(\eta_{10}=194 \mathrm{mV}\right)$ established the synergistic effect in NV/NRGO2 $\left(\eta_{10}=43 \mathrm{mV}\right)$. Furthermore, control experiments were also performed on $\mathrm{NiO}$ and $\mathrm{V}_{2} \mathrm{O}_{5}$ to evaluate their performance in HER (Fig. S7 $\dagger$ ). It was observed that $\mathrm{NiO}\left(\eta_{10}=200 \mathrm{mV}\right)$ and $\mathrm{V}_{2} \mathrm{O}_{5}\left(\eta_{10}=163 \mathrm{mV}\right)$ individually exhibit lower activities compared to $\mathrm{NV}\left(\eta_{10}=97 \mathrm{mV}\right)$ in HER. Therefore, the synergistic contribution of $\mathrm{NiO}$ and $\mathrm{V}_{2} \mathrm{O}_{5}$ in addition to that of NV and NRGO as stated earlier can account for the improved hydrogen evolution ability owing to the synergistic effect in NV/NRGO2. It was noted that NV/NRGO1 showed a slightly better performance compared to $\mathrm{NV}$ in the low overpotential region although its performance deteriorated at higher overpotentials (Table S3 $\dagger$ ). This phenomenon could be attributed to the low exfoliation of NRGO as well as the aggregation and surface blockage of the $\mathrm{Ni}_{3} \mathrm{~V}_{2} \mathrm{O}_{8}$ particles in $\mathrm{NV} /$ NRGO1. Alternatively, the possibility of a less number of active sites coming in contact with the electrolyte as a result of the faster rate of hydrogen evolution at the high overpotential regions could not be overruled. In contrast, $\mathrm{NV} / \mathrm{NRGO} 2$ loaded with $5.6 \mathrm{wt} \%$ of NRGO achieved the lowest value of $\eta_{10}(43 \mathrm{mV})$, demonstrating its superior electrocatalytic HER activity. Such superior HER activity of NV/NRGO2 could be assigned to the uniform distribution of quasi-spherical $\mathrm{Ni}_{3} \mathrm{~V}_{2} \mathrm{O}_{8}$ nanoparticles on NRGO sheets, thereby facilitating a better contact between active sites and the electrolyte $\left(\mathrm{OH}^{-}\right.$ions $){ }^{29}$ However, the $\mathrm{NV} /$ NRGO3 (6.6 wt\%) hybrid exhibited lower electrocatalytic activity due to the restacking of NRGO sheets, self-
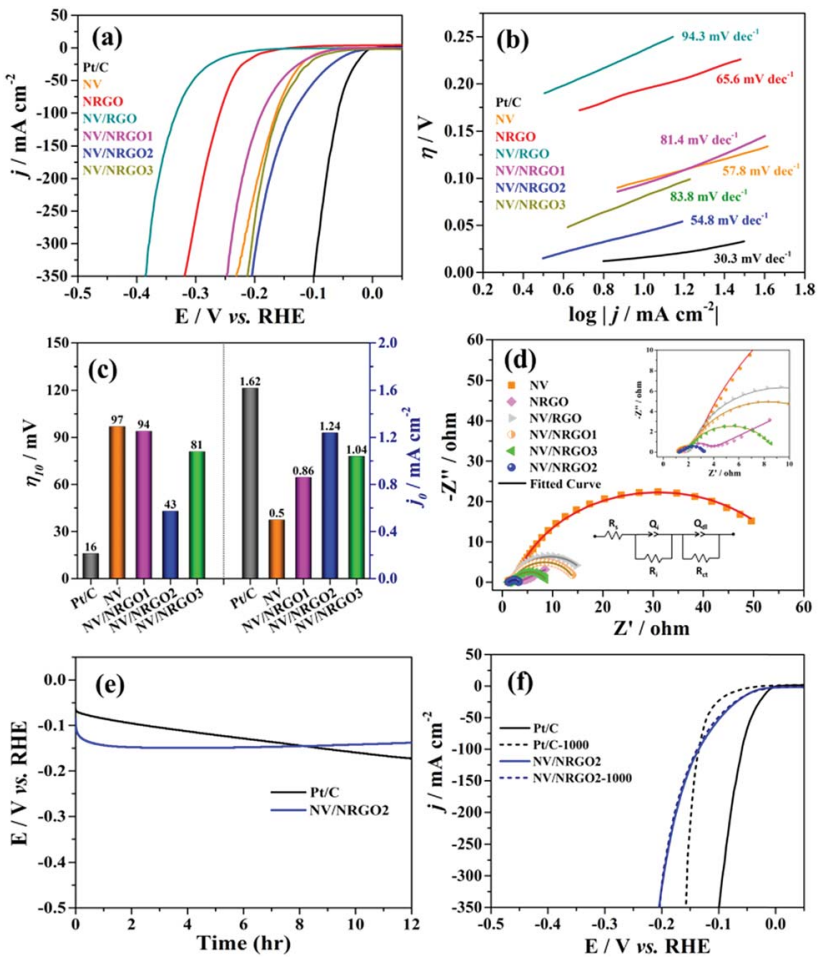

Fig. 4 (a) LSV curves at $5 \mathrm{mV} \mathrm{s}{ }^{-1}$. (b) Tafel plots derived from LSV curves of Pt/C, NV, NRGO, NV/RGO, NV/NRGO1, NV/NRGO2, and NV/ NRGO3. (c) Overpotential $\left(\eta_{10}\right)$ and exchange current density $\left(j_{0}\right)$ of Pt/ C, NV, NV/NRGO1, NV/NRGO2, and NV/NRGO3. (d) Nyquist plots at $\eta$ $=244 \mathrm{mV}$ (equivalent circuit and the magnified plot are shown in the inset) of NV, NRGO, NV/RGO, NV/NRGO1, NV/NRGO2, and NV/ NRGO3. (e) CP curves for $12 \mathrm{~h}$ without iR-correction and (f) initial LSV curves (solid lines) and LSV curves after 1000 cycles (dotted lines) of Pt/ $\mathrm{C}$ and NV/NRGO2. Unless mentioned, all the measurements were carried out with iR-compensation at room temperature in $1.0 \mathrm{M} \mathrm{KOH}$.

agglomeration, and the irregular distribution of catalytic active sites..$^{29}$ Scheme 2 shows the distribution of the active sites in NV/NRGO1, NV/NRGO2, and NV/NRGO3 considering these aspects and the TEM studies discussed earlier. Furthermore, the HER performance of NV/RGO was also compared with that of the NV/NRGO2 hybrid to ascertain the advantages of NRGO if any. Our findings clearly demonstrated the relative inferior performance of $\mathrm{NV} / \mathrm{RGO}\left(\eta_{10}=236 \mathrm{mV}\right)$ due to the poor exfoliation/aggregation of RGO sheets and NV particles, as evident from the FESEM image (Fig. S8b†). In contrast, NV/ NRGO2 displayed a superior HER performance due to the homogeneous distribution of NV particles in the NRGO matrix, as indicated by the TEM image shown in Fig. S5b.† Alternatively, the fact that the presence of enhanced defect sites in NRGO can contribute to the improvement in its HER activity also cannot be ruled out. According to our findings, NV/NRGO2 also outperformed other HER electrocatalysts such as $\mathrm{Ni}_{3}(-$ $\left.\mathrm{VO}_{4}\right)_{2} @ \mathrm{NiCo}_{2} \mathrm{O}_{4} / \mathrm{NF}(113 \mathrm{mV}),{ }^{16} \mathrm{Ni}_{3} \mathrm{~N} @ \mathrm{VN}-\mathrm{NF}(56 \mathrm{mV}),{ }^{30} \mathrm{~N}-\mathrm{Co}-$ $\mathrm{C} / \mathrm{RGO}(130 \mathrm{mV}),{ }^{31} \mathrm{Sr}_{2} \mathrm{RuO}_{4}(61 \mathrm{mV}),{ }^{32} \beta-\mathrm{Ni}(\mathrm{OH})_{2} / \mathrm{Pt}(108 \mathrm{mV}),{ }^{33}$ and nest-like NiCoP $(62 \mathrm{mV})^{34}$ (Table S4†).

A Tafel slope is useful to gain insights into the mechanistic pathway through the rate-determining step (RDS). According to 


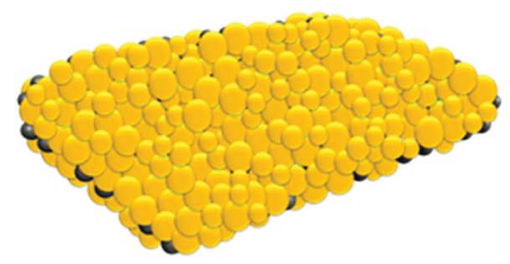

NV/NRGO1

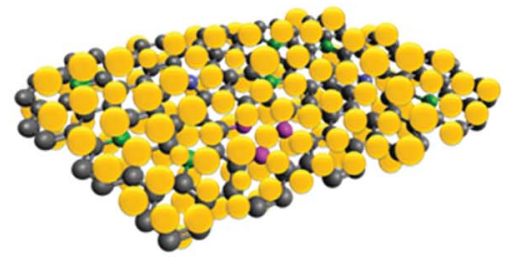

NV/NRGO2

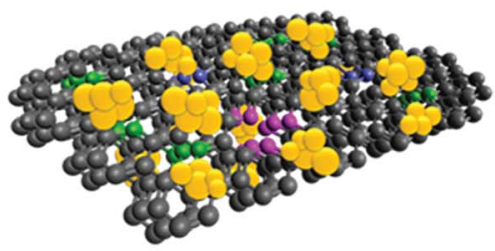

NV/NRGO3



Scheme 2 Distribution of active sites in NV/NRGO hybrids.

the available literature, HER in an alkaline medium proceeds through the Volmer-Heyrovsky or Volmer-Tafel pathways. ${ }^{33}$ In the Volmer step, the dissociation of a surface-adsorbed water molecule results in the formation of surface-adsorbed hydrogen atoms $\left(\mathrm{S}-\mathrm{H}_{\mathrm{ad}}\right)$ and the generation of $\mathrm{OH}^{-}\left(\mathrm{H}_{2} \mathrm{O}+\mathrm{S}+\mathrm{e}^{-} \rightarrow \mathrm{S}-\right.$ $\mathrm{H}_{\mathrm{ad}}+\mathrm{OH}^{-}$). Subsequently, S- $\mathrm{H}_{\mathrm{ad}}$ either combines with anther $\mathrm{H}_{2} \mathrm{O}$ molecule in the Heyrovsky step $\left(\mathrm{H}_{2} \mathrm{O}+\mathrm{S}-\mathrm{H}_{\mathrm{ad}}+\mathrm{e}^{-} \rightarrow \mathrm{H}_{2}+\mathrm{S}\right.$ $+\mathrm{OH}^{-}$) or recombines with another $\mathrm{S}-\mathrm{H}_{\mathrm{ad}}$ to release molecular hydrogen in the Tafel step $\left(2 \mathrm{~S}-\mathrm{H}_{\mathrm{ad}} \rightarrow 2 \mathrm{~S}+\mathrm{H}_{2}\right){ }^{35,36}$ According to the Butler-Volmer kinetics, the rate-determining step can be identified depending on the Tafel slope as the Volmer $(120 \mathrm{mV}$ $\left.\mathrm{dec}^{-1}\right)$, Heyrovsky ( $\left.40 \mathrm{mV} \mathrm{dec}{ }^{-1}\right)$, or Tafel $\left(30 \mathrm{mV} \mathrm{dec}^{-1}\right.$ ) reaction. ${ }^{33,35}$ We observed the Tafel slope (Fig. $4 \mathrm{~b}$ ) values of $\sim 30.3$, $57.8,65.6,94.3,81.4,54.8$, and $83.8 \mathrm{mV} \mathrm{dec}^{-1}$ corresponding to $\mathrm{Pt} / \mathrm{C}, \mathrm{NV}, \mathrm{NRGO}, \mathrm{NV} / \mathrm{RGO}, \mathrm{NV} / \mathrm{NRGO} 1, \mathrm{NV} / \mathrm{NRGO} 2$, and NV/ NRGO3. These findings suggested Tafel and Heyrovsky reactions as the rate determining steps, confirming the overall Volmer-Tafel and Volmer-Heyrovsky pathways in Pt/C and NV/ NRGO2 (Scheme 3), respectively. It was anticipated that the relatively lowest Tafel slope of NV/NRGO2 $\left(54.8 \mathrm{mv} \mathrm{dec}^{-1}\right)$ among those of NV and all its NRGO hybrids, would indicate faster reaction kinetics in HER. It was also noted that the Tafel slope for NV/NRGO2 $\left(54.8 \mathrm{mV} \mathrm{dec}^{-1}\right)$ was lower in comparison to the results of the electrocatalysts studied in the contemporary

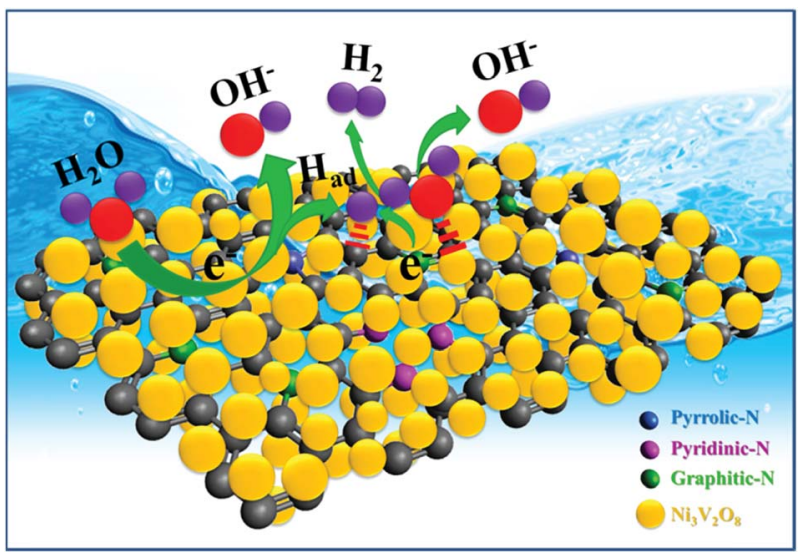

Scheme 3 Volmer-Heyrovsky pathway of hydrogen evolution in an alkaline medium for NV/NRGO2. works reported in the literature such as $\mathrm{NiO} / \mathrm{Ni}-\mathrm{CNT}(84.6 \mathrm{mV}$ $\left.\mathrm{dec}^{-1}\right),{ }^{37} \mathrm{VOOH}$ hollow nanospheres $\left(104 \mathrm{mV} \mathrm{dec}^{-1}\right),{ }^{38} \mathrm{Fe}_{17.5 \%}{ }^{-}$ $\mathrm{Ni}_{3} \mathrm{~S}_{2} / \mathrm{NF}\left(95 \mathrm{mV} \mathrm{dec}{ }^{-1}\right),{ }^{39} \mathrm{P}_{8.6}-\mathrm{Co}_{3} \mathrm{O}_{4} / \mathrm{NF}\left(86 \mathrm{mV} \mathrm{dec}^{-1}\right){ }^{40} \mathrm{Cu}$ NDs/Ni $\mathrm{S}_{2}$ NTs-CFs $\left(76.2 \mathrm{mV} \mathrm{dec}^{-1}\right),{ }^{41}$ and $\mathrm{Ni}_{3} \mathrm{~N} @$ CQDs $(108 \mathrm{mV}$ $\left.\operatorname{dec}^{-1}\right)^{42}$ for HER in an alkaline medium.

Moreover, exchange current density $\left(j_{0}\right)$ is an important parameter to assess the intrinsic electrocatalytic activity of a catalyst. In this regard, the Tafel plot was extrapolated ${ }^{43}$ to $\eta=$ 0 and the $j_{0}$ values of $\mathrm{NV}\left(0.05 \mathrm{~mA} \mathrm{~cm}{ }^{-2}\right), \mathrm{NV} / \mathrm{NRGO} 1(0.86 \mathrm{~mA}$ $\mathrm{cm}^{-2}$ ), NV/NRGO2 $\left(1.24 \mathrm{~mA} \mathrm{~cm}{ }^{-2}\right.$ ) and NV/NRGO3 (1.04 mA $\mathrm{cm}^{-2}$ ) were evaluated, as shown in Fig. 4c. The excellent HER activity of NV/NRGO2 is reflected from its $j_{0}$ value, which is closer to that of $\mathrm{Pt} / \mathrm{C}\left(1.62 \mathrm{~mA} \mathrm{~cm}{ }^{-2}\right)$. Furthermore, the intrinsic activity of an electrocatalyst can be correlated to the turnover frequency (TOF). Accordingly, the TOF values at $\eta=100 \mathrm{mV}$ follow the order NV/NRGO2 $\left(0.021 \mathrm{~s}^{-1}\right)>\mathrm{NV}\left(0.013 \mathrm{~s}^{-1}\right)>\mathrm{NV} /$ NRGO1 $\left(0.008 \mathrm{~s}^{-1}\right)>\mathrm{NV} / \mathrm{NRGO}\left(0.004 \mathrm{~s}^{-1}\right)$. The highest TOF value of NV/NRGO2 further confirmed our earlier contention on its superior intrinsic HER activity. The electrochemical active surface area (ECSA) is an important parameter for the surface redox reactions at the electrode. ${ }^{44}$ Accordingly, the obtained ECSA values (ESI $\dagger$ ) follow the order (Fig. S11b $\dagger$ ) NV $\left(92.5 \mathrm{~cm}^{2}\right)<$ NV/NRGO1 $\left(297.5 \mathrm{~cm}^{2}\right)<$ NV/NRGO3 $\left(455.0 \mathrm{~cm}^{2}\right)<$ NV/NRGO2 $\left(517.5 \mathrm{~cm}^{2}\right)$. These findings clearly suggest that NV/NRGO2 consists of a large number of active sites owing to its highest ECSA compared to all other synthesized electrocatalysts. The Nyquist plots $(\eta=244 \mathrm{mV})$ of NV, NRGO, NV/RGO, NV/NRGO1, NV/NRGO2, and NV/NRGO3 in Fig. 4d demonstrate the corresponding $R_{\mathrm{ct}}$ values of $\sim 57.1,2.2,15.4,12.6,1.6$, and $6.9 \Omega$. The high electronic conductivity of the NRGO sheets accounted for the significantly decreased $R_{\mathrm{ct}}$ value for the NV/NRGO hybrids in comparison to that for NV. Furthermore, the remarkably low $R_{\mathrm{ct}}$ value $(1.6 \Omega)$ of NV/NRGO2 also supported its highly enhanced HER activity.

The long-term stability and the durability of an electrocatalyst are considered to be the most important criteria for its practical applications. ${ }^{45,46}$ In view of this, $\mathrm{Pt} / \mathrm{C}$ and NV/NRGO2 were investigated by the chronopotentiometry (CP) technique (without iR-compensation) at $10 \mathrm{~mA} \mathrm{~cm}{ }^{-2}$ for their long-term durability $(12 \mathrm{~h})$; potential $(E)$ versus time $(t)$ is displayed in Fig. 4e. Our findings demonstrated the appearance of three regions in the $E v$ s. $t$ plot of NV/NRGO2: $92 \mathrm{mV}(t=0)$ to $149 \mathrm{mV}$ 
$(t=2.3 \mathrm{~h}), 149 \mathrm{mV}(t=2.3 \mathrm{~h})$ to $149 \mathrm{mV}(t=5.6 \mathrm{~h})$ and $149 \mathrm{mV}(t$ $=5.6 \mathrm{~h})$ to $137 \mathrm{mV}(t=12 \mathrm{~h})$. This clearly showed that the increase in $E$ was more pronounced in the beginning up to $2.3 \mathrm{~h}$, followed by an almost constant $E$ up to $5.6 \mathrm{~h}$. Thereafter, $E$, in contrast to that of the $\mathrm{Pt} / \mathrm{C}$ electrode, decreased from 149 to $137 \mathrm{mV}$, corresponding to the increase in time from 5.6 to $12 \mathrm{~h}$. A similar behaviour was also noticed for $\mathrm{NiCo}_{2} \mathrm{~S}_{4} / \mathrm{NF}^{47}$ and $\mathrm{CoS} \mid \mathrm{P} / \mathrm{CNT}^{48}$ In contrast to the result for NV/NRGO2, the poor long-term durability of $\mathrm{Pt} / \mathrm{C}$ in $1.0 \mathrm{M} \mathrm{KOH}$ was evident from the continuous increase in the overpotential from $67 \mathrm{mV}$ to $172 \mathrm{mV}$. Subsequently, accelerated stability of the electrocatalysts was achieved by subjecting them to $1000 \mathrm{CV}$ cycles at a scan rate of $100 \mathrm{mV} \mathrm{s}^{-1}$. The corresponding polarization curves (iRcompensated) of $\mathrm{Pt} / \mathrm{C}$ and NV/NRGO2 (Fig. 4f) exhibited an almost unchanged $\eta_{10}$ value for $\mathrm{NV} / \mathrm{NRGO} 2$ in contrast to the increase in $\eta_{10}$ by $59 \mathrm{mV}$ for Pt/C. Therefore, NV/NRGO2 demonstrated much better long-term and accelerated stability in comparison to $\mathrm{Pt} / \mathrm{C}$ in alkaline HER. Further investigations were carried out to study the performance of NV/NRGO2 as a water-splitting electrocatalyst for a real alkaline $(1.0 \mathrm{M} \mathrm{KOH})$ electrolyzer in a two-electrode system (NV/NRGO2 $\|$ NV/NRGO2) (Fig. S12 $\dagger$ ). It was noted that a current density value of $10 \mathrm{~mA}$ $\mathrm{cm}^{-2}$ was achieved in the cell at $1.77 \mathrm{~V}$, which was found to be lower than that reported in previously published works on $\mathrm{Ni}(\mathrm{OH})_{2} / \mathrm{NF} \| \mathrm{Ni}(\mathrm{OH})_{2} / \mathrm{NF}(1.82 \mathrm{~V})^{49}$ and commercial water electrolyzers $(1.8$ to $2.0 \mathrm{~V}) .{ }^{49}$

The structural stability of NV/NRGO2 after the durability tests was studied by XRD, FESEM and Raman spectroscopy; the corresponding findings are displayed in Fig. S13.† The XRD patterns of NVNRGO2 before and after HER are compared in Fig. S13(i). $\dagger$ The appearance of prominent peaks at $35.1^{\circ}$ (122), $43.0^{\circ}(151)$, and $62.5^{\circ}(004)$ is noted in the XRD pattern of NVNRGO2 after electrocatalysis (Fig. S13(ib) $\dagger$ ), corresponding to the orthorhombic phase of $\mathrm{Ni}_{3} \mathrm{~V}_{2} \mathrm{O}_{8}$ (JCPDS no. 74-1484). In addition, the appearance of the (002) peak at $23.1^{\circ}$ can be ascribed to the presence of graphitic carbon in NRGO. Alternatively, the possibility of a peak appearing due to carbon black used in the preparation of the working electrode also cannot be ruled out. An additional low intense peak also appeared at $11.3^{\circ}$, which could be assigned to the (003) plane of NiOOH (JCPDS no. 06-0075) due to the continuous electrolysis of NV/NRGO2 in the alkaline medium. ${ }^{16}$ Furthermore, the Raman spectrum of the sample (after HER) remains more or less unaltered except for the slightly reduced $I_{\mathrm{D}} / I_{\mathrm{G}}$ ratio $(0.82)$ with respect to that for pristine $\mathrm{NV} / \mathrm{NRGO} 2$. This could be attributed to the decrease in the density of the defects in NV/NRGO2 after the HER durability test. The XPS analysis of NV/NRGO2 was also performed after the stability test and the corresponding findings are displayed in Fig. S14. $\uparrow$ The XPS survey scan of the post-HER sample demonstrated the presence of $\mathrm{Ni}, \mathrm{V}, \mathrm{O}, \mathrm{C}$, and $\mathrm{N}$ similar to that for the fresh catalyst. The high-resolution Ni $2 p$ spectrum of the sample after the stability test shows negligible shifts in $\mathrm{Ni} 2 \mathrm{p}_{1 / 2}$ $(872.9 \mathrm{eV})$ and $\mathrm{Ni} 2 \mathrm{p}_{3 / 2}(855.0 \mathrm{eV})$ peaks compared to the spectrum of the sample before HER. In contrast, two additional peaks appear at 873.5 and $856.2 \mathrm{eV}$ in the deconvoluted spectrum of Ni 2p. This can be ascribed to the formation of $\mathrm{NiOOH}$ owing to the surface oxidation of the catalyst by adsorbed $\mathrm{OH}^{-}$ ions as a result of prolonged use in a strong alkaline medium. ${ }^{\mathbf{1 6}}$ Similarly, the new peaks observed in the $\mathrm{V} 2 \mathrm{p}$ region at 521.8 and $515.2 \mathrm{eV}$ can be assigned to $\mathrm{VOOH}^{38}$ In addition, the $\mathrm{O} 1 \mathrm{~s}$ spectrum shows peaks at 531.1, 530.2, and 528.6 eV due to $\mathrm{H}_{2} \mathrm{O}$, $\mathrm{O}-\mathrm{H}$, and the metal-oxygen bond, respectively. ${ }^{38}$ In addition, the comparison of the FESEM images of NV/NRGO2 before (Fig. 3c) and after (Fig. S13(ii) $\dagger$ ) HER shows some changes in the morphology due to the presence of carbon black and the polymeric binder (PVDF) used in electrode preparation. All these findings further strengthen our earlier inference: NV/NRGO2 is a suitable electrocatalyst for practical applications.

The superior HER activity of NV/NRGO2 to those of other electrocatalysts may be ascribed to the synergistic effect of $\mathrm{Ni}_{3} \mathrm{~V}_{2} \mathrm{O}_{8}$ particles with NRGO sheets, uniform particle distribution, $\mathrm{N}$ doping, and the optimised NRGO content. Alternatively, the remarkable increase in the charge transportation of NRGO due to high electrical conductivity also cannot be ruled out. Furthermore, the higher ECSA value of NVNRGO2 $\left(517.5 \mathrm{~cm}^{2}\right)$ most likely originates from the better exposure of the electrolyte to the active sites. Additionally, NRGO also imparted structural stability in its long-term application, as evidenced by post-HER characterizations. Therefore, $\mathrm{NV} / \mathrm{NRGO} 2$ can act as an effective and stable electrocatalyst in an alkaline medium for the hydrogen evolution reaction.

Our findings clearly showed that NV/NRGO2 exhibited superior durability in contrast to other electrochemical properties compared to commercial Pt/C. Most likely, Pt/C facilitates the exposure of relatively more active sites to enable rapid electron and mass transfer. ${ }^{50}$ Similar HER activity could be achieved for NV/NRGO2 by generating more active sites via tuning its morphology or by the modification of the electronic structure by doping. ${ }^{35}$ According to the available literature, an effective alkaline HER electrocatalyst should have the ability of efficient water dissociation at the surface and proper binding with hydrogen intermediates. ${ }^{35,51}$ This could be facilitated by adding another active component on the surface of NV/NRGO2 to achieve similar/better HER activity to/than that of $\mathrm{Pt} / \mathrm{C}$ in an alkaline medium. ${ }^{52-54}$

In summary, an $\mathrm{Ni}_{3} \mathrm{~V}_{2} \mathrm{O}_{8}$ /NRGO hybrid with $5.6 \mathrm{wt} \% \mathrm{NRGO}$ content was successfully fabricated through refluxing and employed as an electrocatalyst for HER in an alkaline medium (1.0 M KOH). The superior performance of the $\mathrm{Ni}_{3} \mathrm{~V}_{2} \mathrm{O}_{8} / \mathrm{NRGO}$ (5.6 wt\%) hybrid manifested a very low overpotential $(\sim 43 \mathrm{mV})$, small Tafel slope $\left(\sim 54.8 \mathrm{mV} \mathrm{dec}{ }^{-1}\right)$, and quite high exchange current density $\left(\sim 1.24 \mathrm{~mA} \mathrm{~cm}{ }^{-2}\right)$ for hydrogen evolution in 1.0 $\mathrm{M} \mathrm{KOH}$. Furthermore, the value of the Tafel slope suggested that HER proceeded through the Volmer-Heyrovsky mechanism. In addition, this hybrid electrocatalyst also demonstrated good long-term and accelerated stability in a strong alkaline solution. Therefore, $\mathrm{Ni}_{3} \mathrm{~V}_{2} \mathrm{O}_{8} / \mathrm{NRGO}$ (5.6 wt $\%$ ) can be used as a low-cost, highly efficient, and stable alternative to the commercial Pt/C electrocatalyst for the industrial production of hydrogen through alkaline water electrolysis.

\section{Conflicts of interest}

There are no conflicts of interest to declare. 


\section{Acknowledgements}

S. K. S. is grateful to IIT Kharagpur for providing a grant for the $\mathrm{CH}$ Instruments and other necessary facilities in this work. A. K. gratefully acknowledges IIT Kharagpur for providing financial support. Authors are also very much thankful to Prof. S. K. Srivastava, Department of Physics, IIT Kharagpur and Prof. M. M. Ghangrekar, School of Environmental Science and Engineering, IIT Kharagpur for XPS and ICP-MS analyses respectively.

\section{References}

1 J. A. Turner, Science, 2004, 305, 972-974.

2 O. Schmidt, A. Gambhir, I. Staffell, A. Hawkes, J. Nelson and S. Few, Int. J. Hydrogen Energy, 2017, 42, 30470-30492.

3 L. Xie, Q. Liu, X. Shi, A. M. Asiri, Y. Luo and X. Sun, Inorg. Chem. Front., 2018, 5, 1365-1369.

4 N. Roy, K. T. Leung and D. Pradhan, J. Phys. Chem. C, 2015, 119, 19117-19125.

5 C. Tang, L. Gan, R. Zhang, W. Lu, X. Jiang, A. M. Asiri, X. Sun, J. Wang and L. Chen, Nano Lett., 2016, 16, 6617-6621.

6 M. Zang, N. Xu, G. Cao, Z. Chen and J. Cui, ACS Catal., 2018, 8, 5062-5069.

7 J. Zhang, B. Yuan, S. Cui, N. Zhang, J. Wei, X. Wang, D. Zhang, R. Zhang and Q. Huo, Dalton Trans., 2017, 46, 3295-3302.

8 C. Zhou, S. Fan, M. Hu, J. Lu, J. Li, Z. H. Huang, F. Kang and R. Lv, J. Mater. Chem. A, 2017, 5, 15517-15524.

9 M. Xing, L. B. Kong, M. C. Liu, L. Y. Liu, L. Kang and Y. C. Luo, J. Mater. Chem. A, 2014, 2, 18435-18443.

10 Y. Zhao, Y. Liu, X. Du, R. Han and Y. Ding, J. Mater. Chem. A, 2014, 2, 19308-19314.

11 L. Liardet and X. Hu, ACS Catal., 2018, 8, 644-650.

12 C. Wang, D. Fang, H. Wang, Y. Cao, W. Xu, X. Liu, Z. Luo, G. Li, M. Jiang and C. Xiong, Sci. Rep., 2016, 6, 4-11.

13 R. Kumar, P. Rai and A. Sharma, J. Mater. Chem. A, 2016, 4, 9822-9831.

14 D. Nandi, M. Gnanaseelan, F. Simon and J. Pionteck, New J. Chem., 2017, 41, 5620-5627.

15 R. Kumar, P. K. Gupta, P. Rai and A. Sharma, New J. Chem., 2018, 42, 1243-1249.

16 X. Shang, J. Q. Chi, S. S. Lu, B. Dong, Z. Z. Liu, K. L. Yan, W. K. Gao, Y. M. Chai and C. G. Liu, Electrochim. Acta, 2017, 256, 100-109.

17 B. Chang, G. Zhao, Y. Shao, L. Zhang, B. Huang, Y. Wu and X. Hao, J. Mater. Chem. A, 2017, 5, 18038-18043.

18 S. K. Srivastava and J. Pionteck, J. Nanosci. Nanotechnol., 2015, 15, 1984-2000.

19 B. M. Bhanage, S. Fujita, Y. Ikushima and M. Arai, Green Chem., 2003, 5, 429-432.

20 H. Liu, J. Zhang, D. Xu, B. Zhang, L. Shi, L. Huang and S. Tan, Appl. Surf. Sci., 2014, 317, 370-377.

21 G. Gao, S. Lu, B. Dong, Y. Xiang, K. Xi and S. Ding, J. Mater. Chem. A, 2016, 4, 6264-6270.

22 G. Liu, L. Wang, B. Wang, T. Gao and D. Wang, RSC Adv., 2015, 5, 63553-63560.
23 A. A. Dubale, W. N. Su, A. G. Tamirat, C. J. Pan, B. A. Aragaw, H. M. Chen, C. H. Chen and B. J. Hwang, J. Mater. Chem. A, 2014, 2, 18383-18397.

24 S. K. Srivastava and K. Manna, J. Phys. Chem. C, 2017, 121, 18214-18220.

25 M. Sakthivel, R. Sukanya, S. M. Chen and K. C. Ho, J. Mater. Chem. A, 2019, 7, 12565-12581.

26 S. Lu, T. Zhu, Z. Li, Y. Pang, L. Shi, S. Ding and G. Gao, J. Mater. Chem. A, 2018, 6, 7005-7013.

27 C. Zhou, S. Fan, M. Hu, J. Lu, J. Li, Z. H. Huang, F. Kang and R. Lv, J. Mater. Chem. A, 2017, 5, 15517-15524.

28 M. J. Gómez, A. Loiácono, L. A. Pérez, E. A. Franceschini and G. I. Lacconi, ACS Omega, 2019, 4, 2206-2216.

29 F. C. Shen, Y. Wang, Y. J. Tang, S. L. Li, Y. R. Wang, L. Z. Dong, Y. F. Li, Y. Xu and Y. Q. Lan, ACS Energy Lett., 2017, 2, 1327-1333.

30 P. Zhou, B. Huang, P. Wang, X. Qin, D. Xing, X. Zhang, Y. Liu, Z. Wang, Z. Zheng and Y. Dai, J. Mater. Chem. A, 2019, 7, 5513-5521.

31 S. Palani, C. C. Liao, W. F. Chen, T. Chou, I. Shown, A. Sabbah, Y. G. Lin, J. F. Lee, M. K. Tsai, K. H. Chen and L. C. Chen, J. Mater. Chem. A, 2019, 7, 7179-7185.

32 Y. Zhu, H. A. Tahini, Z. Hu, J. Dai, Y. Chen, H. Sun, W. Zhou, M. Liu, S. C. Smith, H. Wang and Z. Shao, Nat. Commun., 2019, 10, 149.

33 X. Yu, J. Zhao, L. R. Zheng, Y. Tong, M. Zhang, G. Xu, C. Li, J. Ma and G. Shi, ACS Energy Lett., 2018, 3, 237-244.

34 C. Du, L. Yang, F. Yang, G. Cheng and W. Luo, ACS Catal., 2017, 7, 4131-4137.

35 N. Mahmood, Y. Yao, J. W. Zhang, L. Pan, X. Zhang and J. J. Zou, Adv. Sci., 2018, 5, 1700464, DOI: 10.1002/ advs.201700464.

36 M. Gong, D. Y. Wang, C. C. Chen, B. J. Hwang and H. Dai, Nano Res., 2016, 9, 28-46.

37 M. Gong, W. Zhou, M. C. Tsai, J. Zhou, M. Guan, M. C. Lin, B. Zhang, Y. Hu, D. Y. Wang, J. Yang, S. J. Pennycook, B. J. Hwang and H. Dai, Nat. Commun., 2014, 5, 4695.

38 H. Shi, H. Liang, F. Ming and Z. Wang, Angew. Chem., Int. Ed., 2017, 56, 573-577.

39 G. Zhang, Y. S. Feng, W. T. Lu, D. He, C. Y. Wang, Y. K. Li, X. Y. Wang and F. F. Cao, ACS Catal., 2018, 8, 5431-5441.

40 Z. Wang, H. Liu, R. Ge, X. Ren, J. Ren, D. Yang, L. Zhang and X. Sun, ACS Catal., 2018, 8, 2236-2241.

41 J. X. Feng, J. Q. Wu, Y. Tong and G. R. Li, J. Am. Chem. Soc., 2018, 140, 610-617.

42 M. Zhou, Q. Weng, Z. I. Popov, Y. Yang, L. Y. Antipina, P. B. Sorokin, X. Wang, Y. Bando and D. Golberg, ACS Nano, 2018, 12, 4148-4155.

43 Y. Y. Chen, Y. Zhang, W. J. Jiang, X. Zhang, Z. Dai, L. J. Wan and J. S. Hu, ACS Nano, 2016, 10, 8851-8860.

44 X. Li, P. F. Liu, L. Zhang, M. Y. Zu, Y. X. Yang and H. G. Yang, Chem. Commun., 2016, 52, 10566-10569.

45 A. Karmakar and S. K. Srivastava, ACS Appl. Mater. Interfaces, 2017, 9, 22378-22387.

46 T. Zhang, M. Y. Wu, D. Y. Yan, J. Mao, H. Liu, W. Bin Hu, X. W. Du, T. Ling and S. Z. Qiao, Nano Energy, 2018, 43, 103-109. 
47 L. Ma, Y. Hu, R. Chen, G. Zhu, T. Chen, H. Lv, Y. Wang, J. Liang, H. Liu, C. Yan, H. Zhu, Z. Tie, Z. Jin and J. Liu, Nano Energy, 2016, 24, 139-147.

48 W. Liu, E. Hu, H. Jiang, Y. Xiang, Z. Weng, M. Li, Q. Fan, X. Yu, E. I. Altman and H. Wang, Nat. Commun., 2016, 7, 1-9.

49 L. Jingshan, I. Jeong-Hyeok, M. T. Mayer, S. Marcel, N. Mohammad Khaja, P. Nam-Gyu, T. S David, F. H. Jin and G. Michael, Science, 2014, 345, 1593-1596.
50 D. Wang, C. Han, Z. Xing, Q. Li and X. Yang, J. Mater. Chem. A, 2018, 6, 15558-15563.

51 J. Wang, T. Qiu, X. Chen, Y. Lu and W. Yang, J. Power Sources, 2015, 293, 178-186.

52 M. Zeng and Y. Li, J. Mater. Chem. A, 2015, 3, 14942-14962.

53 N. Danilovic, R. Subbaraman, D. Strmcnik, K. C. Chang, A. P. Paulikas, V. R. Stamenkovic and N. M. Markovic, Angew. Chem., Int. Ed., 2012, 51, 12495-12498.

54 Z. Liang, H. S. Ahn and A. J. Bard, J. Am. Chem. Soc., 2017, 139, 4854-4858. 